\title{
Evidence for horizon-scale power from CMB polarization
}

\author{
Michael J. Mortonson 1,2 , , and Wayne $\mathrm{Hu}^{2,3}$ \\ ${ }^{1}$ Department of Physics, University of Chicago, Chicago IL 60637 \\ ${ }^{2}$ Kavli Institute for Cosmological Physics and Enrico Fermi Institute, \\ University of Chicago, Chicago IL 60637, U.S.A. \\ ${ }^{3}$ Department of Astronomy 8 Astrophysics, University of Chicago, Chicago IL 60637
}

(Dated: March 24, 2022)

\begin{abstract}
The CMB temperature power spectrum offers ambiguous evidence for the existence of horizonscale power in the primordial power spectrum due to uncertainties in spatial curvature and the physics of cosmic acceleration as well as the observed low quadrupole. Current polarization data from WMAP provide evidence for horizon-scale power that is robust to these uncertainties. Polarization on the largest scales arises mainly from scattering at $z \lesssim 6$ when the universe is fully ionized, making the evidence robust to ionization history variations at higher redshifts as well. A cutoff in the power spectrum is limited to $C=k_{C} / 10^{-4} \mathrm{Mpc}^{-1}<5.2(95 \%$ CL) by polarization, only slightly weaker than joint temperature and polarization constraints in flat $\Lambda \mathrm{CDM}(C<4.2)$. Planck should improve the polarization limit to $C<3.6$ for any model of the acceleration epoch and ionization history as well as provide tests for foreground and systematic contamination.
\end{abstract}

\section{INTRODUCTION}

Whether or not the CMB temperature power spectrum provides evidence for horizon-scale power in the primordial power spectrum depends on assumptions about spatial curvature and the physics of late-time cosmic acceleration. In the flat $\Lambda \mathrm{CDM}$ cosmology, roughly half of the power at the largest angular scales is contributed by the integrated Sachs-Wolfe (ISW) effect from the decay of the potential during the acceleration epoch. Due to projection effects, these contributions primarily come from fluctuations on scales smaller than a tenth of the current horizon.

Indeed, the large ISW effect presents a challenge for explanations of the observed low CMB temperature quadrupole in terms of the primordial power spectrum [1, 2, 3]. Nevertheless, this feature has motivated many studies of models in which the usual, nearly scaleinvariant, inflationary power spectrum is modified by a cutoff that suppresses large-scale power 4, 5, 6, 7, 8, 9, 10, 11, 12, 13, 14, 15, 16, 17, 18, 19, 20, 21]. In a flat $\Lambda \mathrm{CDM}$ context, such models can marginally improve the fit to temperature data over that of a power-law spectrum by removing power on scales $k \lesssim 3 \times 10^{-4} \mathrm{Mpc}^{-1}$. The ISW effect prevents a more substantial improvement from a horizon-scale cutoff. Models that suppress power on smaller scales are disfavored by the data since the observed power in CMB temperature at $\ell \gtrsim 4$ is consistent with the predictions of the power-law spectrum.

These CMB temperature-based conclusions depend strongly on the model for late time acceleration. For example, variations in the equation of state of the dark energy, spatial curvature, and dark energy clustering can change the ISW contributions at low multipoles [22, 23]. In more exotic modified gravity models, a

*Electronic address: mjmort@uchicago.edu horizon-scale cutoff can actually be strongly favored. In the self-accelerating braneworld model [24], a cutoff at $k \sim 8 \times 10^{-4} \mathrm{Mpc}^{-1}$ is preferred by the temperature data [25].

CMB polarization, on the other hand, is free of the ISW effect but retains the sensitivity to a large-scale cutoff [10, 26, 27, 28]. In this paper, we demonstrate the robustness of polarization inferences about horizonscale cutoffs in power by comparing the constraints from temperature and polarization using both current (5-year) CMB data from WMAP [29, 30, 31] and forecasts for data from Planck [32]. We describe the fiducial cutoff model and illustrate the degeneracies with the ISW effect in Sec. [I]. We show how CMB polarization constraints on the cutoff model are free from these degeneracies as well as ionization history uncertainties in Sec. III and we discuss our conclusions in Sec. IV]

\section{HORIZON-SCALE POWER}

For definiteness, we model the primordial curvature power spectrum with an exponential cutoff at $k<k_{C}$,

$$
\Delta_{\zeta}^{2}=A_{s}\left[1-e^{-\left(k / k_{C}\right)^{\alpha}}\right]\left(\frac{k}{k_{0}}\right)^{n_{s}-1},
$$

motivated by a transition between stages of inflation [7]. Here $A_{s}$ is the normalization of the power spectrum at a pivot scale of $k_{0}=0.05 \mathrm{Mpc}^{-1}$ and $n_{s}$ is the spectral tilt. We take $\alpha=3.35$ as in Ref. 7], but the CMB power spectra are insensitive to the exact value of this parameter [16]. For notational convenience, we define

$$
k_{C}=C \times 10^{-4} \mathrm{Mpc}^{-1} \approx 0.3 C H_{0} / h,
$$

such that $C$ is of order unity for cutoff scales on the horizon.

A reduction of large scale power from a finite cutoff $C>0$ can be partially compensated by the ISW effect from the late-time decay of gravitational potentials. 


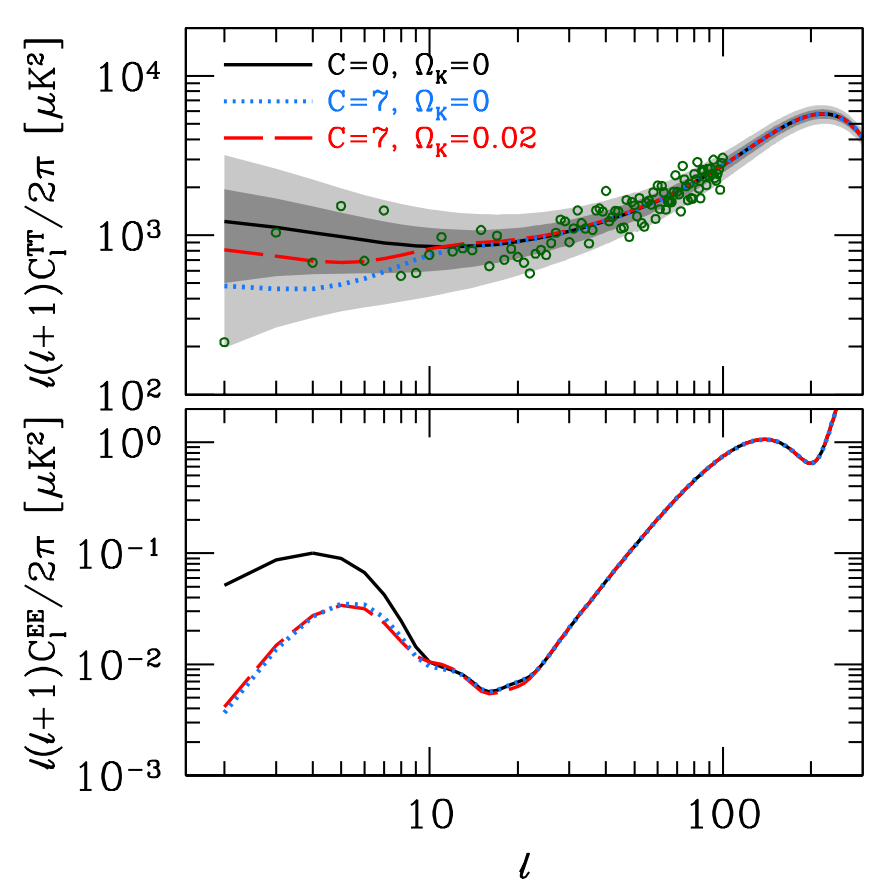

FIG. 1: The cutoff-curvature degeneracy in the CMB TT spectrum (top) is broken in the EE spectrum (bottom). Solid curves show a flat model with no large-scale cutoff $(C=0$, with $68 \%$ and $95 \%$ CL cosmic variance bands per $\ell$ for $T T$ ); other curves have a cutoff at $C=7$. Open circles show WMAP temperature data at $\ell \leq 100$. The WMAP measurement errors are not plotted since they are smaller than the point symbols. For the flat models, $h=0.724$, while the open model with $\Omega_{\mathrm{K}}=0.02$ has $h=0.90$ to preserve the $\mathrm{CMB}$ acoustic scale. All other parameters are the same for the 3 models.

For example, negative spatial curvature can substantially boost power at large angular scales. Figure 1 illustrates the degeneracy between the scale of the exponential cutoff and spatial curvature through the ISW effect. The polarization spectrum, on the other hand, does not receive contributions from the ISW effect and better reveals the presence of a cutoff.

With WMAP data, the flat model with a cutoff at $C=7$ in Fig. 1 can be distinguished from the flat $C=0$ model at high significance using temperature data alone. The likelihood ratio statistic gives $-2 \Delta \ln \mathcal{L}_{T T}=11.7$ relative to the model with no cutoff. However, the open model with a cutoff is more difficult to distinguish from the flat $C=0$ model using only temperature $\left(-2 \Delta \ln \mathcal{L}_{T T}=3.1\right)$. With the addition of polarization data, the open model becomes distinguishable with $-2 \Delta \ln \mathcal{L}_{\text {tot }}=12.6$. This example suggests that even with current data, polarization can provide comparable constraints to temperature in a manner that is robust to curvature and the physics of the acceleration epoch.

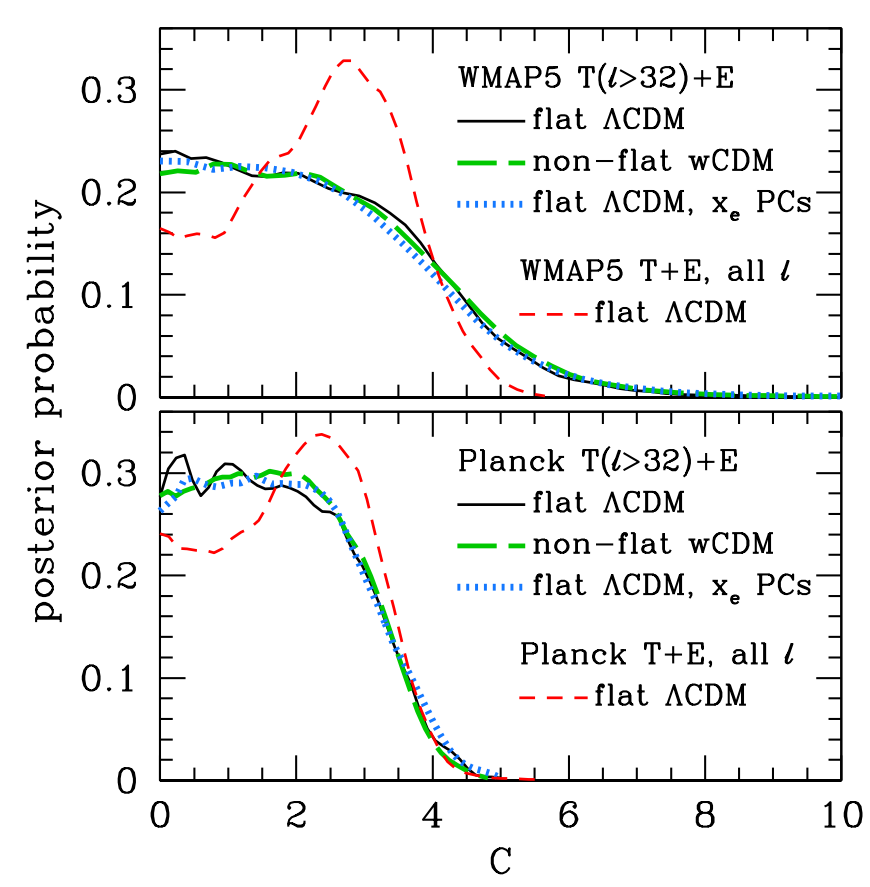

FIG. 2: Marginalized posterior probability for the cutoff scale $C$ using WMAP (top panel) and simulated Planck data (bottom panel), showing robustness of the polarization constraint to curvature, dark energy, and the ionization history. For Planck, the simulated spectra are constrained to the WMAP temperature measurements at $\ell \leq 100$. (See text for details.)

\section{ROBUST POLARIZATION CONSTRAINTS}

To quantify the constraints on horizon-scale power from polarization, test their robustness, and examine their potential for future measurements, we adopt a Markov Chain Monte Carlo (MCMC) approach. We perform this analysis using modified versions of CAMB [33], CosmoMC 34, 35], and the 5-year WMAP likelihood code [30, 36]. The resulting constraints on the cutoff scale $C$ are summarized in Fig. 2 .

We begin with an analysis in the flat $\Lambda \mathrm{CDM}$ cosmology where ISW contributions are nearly fixed by parameters that are well-determined by the acoustic peaks. We add $C$ to the standard set of parameters for MCMC analyses of CMB data, $\left\{\Omega_{b} h^{2}, \Omega_{c} h^{2}, h, \tau, A_{s}, n_{s}\right\}$. As expected, with WMAP data there is a marginal preference for $C \approx 3$ due to the low observed power in the temperature spectrum at low multipoles (see Fig. 2, top panel) [4, 77, 8, 10, 12, 13, 16, 17, 18, 20]. With a flat prior on $C$, the $95 \%$ CL upper bound is $C<4.2$. Without polarization data, this constraint weakens to $C<5.3$.

In the flat $\Lambda \mathrm{CDM}$ context, most of the temperatureonly constraint on $C$ comes from the temperature spectrum at $\ell \lesssim 30$. Consequently, the bound is sensitive to assumptions about the curvature and the dark energy. To test this dependence, we omit the temperature data at $\ell \leq 32$. (This is a convenient dividing point since the 
WMAP likelihood code uses different methods to compute the likelihood at scales above and below $\ell=32[30]$.) With only the temperature data at smaller scales, the upper limit is $C \lesssim 25$, reflecting a near elimination of the constraint. Nonetheless, once polarization is added the bound is only marginally weaker than the full (all $\ell$ ) constraint, $C \lesssim 5.2$ (see Fig. 2, top panel). Thus the polarization constraint is already competitive with the limits from temperature even in the most restrictive flat $\Lambda \mathrm{CDM}$ context. Moreover, the polarization constraint is less model-dependent: in Fig. 2, the posterior probability of $C$ is nearly unchanged even if both curvature and a constant dark energy equation of state $w$ (with prior $-2<w<0$ ) are included as additional MCMC parameters and marginalized. In fact, the constraint from polarization is robust to even more extreme changes such as modified gravity explanations for cosmic acceleration [25].

Polarization constraints are also robust to uncertainties in the ionization history. As noted in Ref. [37], predictions for the lowest few multipoles in polarization are robust to reionization variation since their contributions arise mainly from $z \lesssim 6$ where we know that the universe is fully ionized. The impact of variations at higher redshift from projection effects can be effectively controlled by measurements at higher multipoles where they make most of their contribution.

To quantify this robustness to the ionization history, we adopt the principal components (PCs) technique 38, 39], in which the evolution of the ionization fraction is parametrized as

$$
x_{e}(z)=x_{e}^{\mathrm{fid}}(z)+\sum_{\mu=1}^{N} m_{\mu} S_{\mu}(z),
$$

where $x_{e}^{\mathrm{fid}}(z)$ is an arbitrary fiducial model (taken here to be constant $\left.x_{e}^{\mathrm{fid}}=0.15\right), S_{\mu}(z)$ are the reionization PCs, and the PC amplitudes $m_{\mu}$ are subject to physicality bounds corresponding to $0 \leq x_{e} \leq 1$ as described in Ref. [39]. The PC code modifications to CAMB and CosmoMC have been made publicly available [40]. For a conservative upper limit to the start of reionization of $z_{\max }=30, N=5 \mathrm{PCs}$ are sufficient to completely represent the effects of ionization variation on the CMB polarization power spectrum [39]. Hence marginalizing these parameters makes constraints on $C$ robust to any ionization history for $z<30$. In Fig. 2 (top panel), we show that WMAP constraints on $C$ are almost entirely unchanged by the marginalization over reionization parameters.

In the near future, constraints on horizon-scale power should be dominated by polarization information. We use a simulated temperature and polarization data set to make a forecast for the recently launched Planck satellite. It is important here to account for the fact that WMAP has already measured the temperature power spectrum to the cosmic variance limit at low multipoles (see Fig. 1) and that there is a marginal preference for a finite cut- off. We therefore define the simulated temperature power spectrum for Planck as

$$
\hat{C}_{\ell}^{T T}= \begin{cases}\hat{C}_{\ell}^{T T(\mathrm{WMAP})}, & \ell \leq 100 \\ C_{\ell}^{T T(\mathrm{fid})}, & \ell>100\end{cases}
$$

where $\hat{C}_{\ell}^{T T(\text { WMAP) }}$ is taken from the WMAP data and $C_{\ell}^{T T \text { (fid) }}$ is the temperature power spectrum of the fiducial flat $\Lambda$ CDM model, $\left\{\Omega_{b} h^{2}=0.0224, \Omega_{c} h^{2}=\right.$ $0.108, h=0.724, \tau=0.089, A_{s}=2.137 \times 10^{-9}, n_{s}=$ $0.96\}$. For the polarization data, we take the ensemble mean of the fiducial model given the WMAP temperature constraint

$$
\begin{aligned}
\hat{C}_{\ell}^{E E} & =C_{\ell}^{E E(\text { fid })}\left[1+R_{\ell}^{2}\left(\frac{\hat{C}_{\ell}^{T T}}{C_{\ell}^{T T(\text { fid })}}-1\right)\right], \\
\hat{C}_{\ell}^{T E} & =C_{\ell}^{T E(\text { fid })} \frac{\hat{C}_{\ell}^{T T}}{C_{\ell}^{T T(\text { fid })}},
\end{aligned}
$$

where

$$
R_{\ell}=\frac{C_{\ell}^{T E(\text { fid })}}{\sqrt{C_{\ell}^{T T(\text { fid })} C_{\ell}^{E E(\text { fid })}}}
$$

is the temperature-polarization correlation coefficient in the fiducial model.

For the Planck satellite noise specifications, we take a combination of the central 70, 100, and $143 \mathrm{GHz}$ channels with the sensitivity and resolution given in Ref. [32]. The other Planck frequency channels are effectively used for foreground monitoring and removal as well as checks for systematic effects. The results of the MCMC for Planck are shown in Fig. 2 (bottom panel). Note that now the constraints with and without the $\ell \leq 32$ multipoles in the temperature spectrum are comparable, aside from the slight preference for $C \sim 3$ in the former case, showing that polarization is expected to dominate Planck's constraint on $C$. Like the WMAP polarization constraint, the upper limit $C \lesssim 3.6(95 \% \mathrm{CL})$ from Planck polarization data remains robust to marginalization of the curvature and a constant dark energy equation of state. It is robust to marginalization over the $\mathrm{PC}$ reionization parameters as well.

\section{DISCUSSION}

We have shown that current polarization data from WMAP provide evidence for horizon-scale power that, unlike the evidence from temperature data, is robust to uncertainties in spatial curvature, dark energy, and ionization history variations. A cutoff in the inflationary power spectrum is limited to $C=k_{c} / 10^{-4} \mathrm{Mpc}^{-1}<5.2$ (95\% CL) by polarization. This constraint is only slightly weaker than joint temperature and polarization limits in flat $\Lambda \mathrm{CDM}(C<4.2)$. 
Data from Planck should improve these constraints to the point where polarization dominates the limit for any model of the acceleration epoch and ionization history. Statistical errors should improve to $C<3.6$ if the true model has no cutoff. Perhaps more importantly, the larger frequency coverage of Planck should make the constraint more robust to foregrounds and other systematic effects.
Acknowledgments: This work was supported by NSF PHY-0114422 and NSF PHY-0551142 at the KICP. MJM was additionally supported by the NSF GRFP. WH was additionally supported by DOE contract DE-FG0290ER-40560 and the Packard Foundation.
[1] C. L. Bennett et al., Astrophys. J. 464, L1 (1996), arXiv:astro-ph/9601067.

[2] C. L. Bennett et al., Astrophys. J. Suppl. 148, 1 (2003), arXiv:astro-ph/0302207.

[3] D. N. Spergel et al., Astrophys. J. Suppl. 148, 175 (2003), arXiv:astro-ph/0302209.

[4] Y.-P. Jing and L.-Z. Fang, Phys. Rev. Lett. 73, 1882 (1994), arXiv:astro-ph/9409072.

[5] J. Yokoyama, Phys. Rev. D59, 107303 (1999).

[6] A. Linde, JCAP 0305, 002 (2003), arXiv:astro-ph/0303245.

[7] C. R. Contaldi, M. Peloso, L. Kofman and A. Linde, JCAP 0307, 002 (2003), arXiv:astro-ph/0303636.

[8] S. L. Bridle, A. M. Lewis, J. Weller and G. Efstathiou, Mon. Not. Roy. Astron. Soc. 342, L72 (2003), arXiv:astro-ph/0302306.

[9] G. Efstathiou, Mon. Not. Roy. Astron. Soc. 343, L95 (2003), arXiv:astro-ph/0303127.

[10] J. M. Cline, P. Crotty and J. Lesgourgues, JCAP 0309, 010 (2003), arXiv:astro-ph/0304558.

[11] B. Feng and X. Zhang, Phys. Lett. B570, 145 (2003), arXiv:astro-ph/0305020.

[12] Y.-S. Piao, B. Feng and X.-m. Zhang, Phys. Rev. D69, 103520 (2004), arXiv:hep-th/0310206.

[13] A. Niarchou, A. H. Jaffe and L. Pogosian, Phys. Rev. D69, 063515 (2004), arXiv:astro-ph/0308461.

[14] A. Lasenby and C. Doran, Phys. Rev. D71, 063502 (2005), arXiv:astro-ph/0307311.

[15] M. H. Kesden, M. Kamionkowski and A. Cooray, Phys. Rev. Lett. 91, 221302 (2003), arXiv:astro-ph/0306597.

[16] R. Sinha and T. Souradeep, Phys. Rev. D74, 043518 (2006), arXiv:astro-ph/0511808.

[17] M. Bridges, A. N. Lasenby and M. P. Hobson, Mon. Not. Roy. Astron. Soc. 369, 1123 (2006), arXiv:astro-ph/0511573.

[18] M. Bridges, A. N. Lasenby and M. P. Hobson, Mon. Not. R. Astron. Soc. 381, 68 (2007), arXiv:astro-ph/0607404.

[19] D. Boyanovsky, H. J. de Vega and N. G. Sanchez, Phys. Rev. D74, 123007 (2006), arXiv:astro-ph/0607487.
[20] D. N. Spergel et al., Astrophys. J. Suppl. 170, 377 (2007), arXiv:astro-ph/0603449.

[21] R. K. Jain, P. Chingangbam, J.-O. Gong, L. Sriramkumar and T. Souradeep, JCAP 0901, 009 (2009), arXiv:0809.3915.

[22] W. Hu, Astrophys. J. 506, 485 (1998), arXiv:astro-ph/9801234.

[23] R. Bean and O. Dore, Phys. Rev. D69, 083503 (2004), arXiv:astro-ph/0307100.

[24] G. R. Dvali, G. Gabadadze and M. Porrati, Phys. Lett. B485, 208 (2000), arXiv:hep-th/0005016.

[25] W. Fang et al., Phys. Rev. D78, 103509 (2008), arXiv:0808.2208.

[26] O. Dore, G. P. Holder and A. Loeb, Astrophys. J. 612, 81 (2004), arXiv:astro-ph/0309281.

[27] C. Skordis and J. Silk, arXiv:astro-ph/0402474.

[28] C. Gordon and W. Hu, Phys. Rev. D70, 083003 (2004), arXiv:astro-ph/0406496.

[29] M. R. Nolta et al., Astrophys. J. Suppl. 180, 296 (2009), arXiv:0803.0593.

[30] J. Dunkley et al., Astrophys. J. Suppl. 180, 306 (2009), arXiv:0803.0586.

[31] E. Komatsu et al., Astrophys. J. Suppl. 180, 330 (2009), arXiv:0803.0547.

[32] The Planck Collaboration, arXiv:astro-ph/0604069

[33] A. Lewis, A. Challinor and A. Lasenby, Astrophys. J. 538, 473 (2000), arXiv:astro-ph/9911177.

[34] A. Lewis and S. Bridle, Phys. Rev. D66, 103511 (2002), arXiv:astro-ph/0205436.

[35] http://cosmologist.info/cosmomc/

[36] http://lambda.gsfc.nasa.gov/

[37] M. J. Mortonson and W. Hu, Astrophys. J. Lett. 686, L53 (2008), arXiv:0804.2631.

[38] W. Hu and G. P. Holder, Phys. Rev. D68, 023001 (2003), arXiv:astro-ph/0303400.

[39] M. J. Mortonson and W. Hu, Astrophys. J. 672, 737 (2008), arXiv:0705.1132.

[40] http://background.uchicago.edu/camb_rpc/ 